\title{
DETERMINING THE RELATIONSHIP BETWEEN ALEXITHYMIA, SKIN SENSITIVITY, AND DISGUST
}

\begin{abstract}
Alexithymia represents an individual's inability to recognize their own or someone else's emotions and also the inability to communicate them. It has a documented influence on many cognitive and emotional activities of human life. Skin and touch are two of the primary vehicles for conveying emotions between individuals. It is on account of the connection between these elements that this study has focused on determining more precisely the relation between skin related satisfaction and disgust and alexithymia.

In total 357 students participated in the survey, 286 (82.9\%) were female and the average participant age was $20.54(\min =19 ; \max =26 ; \mathrm{SD}=1.24)$. Apart from this demographical information, the survey consisted of the Skin Satisfaction Questionnaire (TSD-Q 30), the Toronto Alexithymia Questionnaire (TAS-20), and the Disgust Propensity and Sensitivity Questionnaire (DPSS).

Based on an analysis of the survey results, Alexithymia was shown to correlate strongly with the shame aspect of skin satisfaction, but only with TAS-20 factors 1 and 2. A weak correlation was demonstrated between positive disgust and alexithymia. Though only a weak correlation, or no correlations as all, between touch satisfaction and alexithymia was established, touch satisfaction and shame accounted for $21.2 \%$ of alexithymia variance, while disgust measurement scales accounted for only $1.3 \%$.
\end{abstract}

1 Fakultet za pravne i poslovne studije dr Lazar Vrkatić, Bul. Oslobođenja 76, Novi Sad, email: nikola.rokvic@mf.uns.ac.rs 
Out of skin satisfaction parameters, alexithymia appeared to be most strongly connected with shame. Disgust parameters were shown to have a very weak influence on alexithymia variance. Although skin is one of the primary sense organs and touch is capable of conveying emotion, there was found only a weak correlation between, and measured influence of, satisfaction with skin and alexithymia.

\section{Introduction}

Alexithymia represents an individual's inability to recognize their own or someone else's emotions and also the inability to communicate them (Taylor, 1984). It is characterized by four main features: 1. difficulty describing and identifying feelings subjective feelings, 2 . Trouble differentiating between feelings and physical sensations, 3. Limited imaginative processes, 4 . An externally oriented cognitive style (Timoney \& Holder, 2013). The inability or reduced capacity to experience emotion has been proposed as a fifth feature of alexithymia (Bermond, Vorst, Vingerhoets, \& Gerritsen, 1999). One of the features of the inability of individuals with high alexithymia scores to perceive feelings is a decreased ability to perceive the emotions of facial expressions (Grynberg, Corneille, Maurage, Vermeulen, Bertholz, \& Luminet, 2012)

Alexithymia is a well-researched subject and its relationship with many psychological constructs has been extensively documented. Demographically speaking, it has been shown to have higher rates of incidence within populations of the male sex, of lower income, of those with lower degrees of education, and of those living a single lifestyle (Mattila, Poutanen, Koivisto, Salokanges, \& Joukamaa, 2007). Additionally, alexithymia has also been tied to many clinical constructs such as depression (Honkalampi, Hintikka, Tanskanen, Lehtonen, \& Viinamaki, 2000), traumatic brain injury (Koponen, Taiminen, Honkalampi, Joukamaa, Viinamaki, \& Kurki, 2005), posttraumatic stress disorder (Frewen, Dozolis, Neufeld, \& Lanius, 2008), eating disorders (Westwood, Kerr-Gaffney, Stahl, \& Tchanturia, 2017), and many others. As stated previously, there is a relationship between bodily sensation and alexithymia that can result in a positive correlation between palpation pain and alexithymia (Sivik, 1993). A lower awareness of emotional re- 
sponses results in a discrepancy between subjective and physiological responses to emotional or stressful stimuli (Grynberg, Davydov, Vermeulen, \& Luminet, 2012). This same feature is also associated with a distortion of body representations and a lower integration of multisensory inputs (Grynberg \& Pollatos, 2015).

Beyond these attachments, alexithymia has a negative relationship with general well-being (Timoney \& Holder, 2013), and correlates strongly with the Big Five personality traits (Rokvic \& Jovanovic, 2018). In fact, its good temporal invariance has resulted in some authors labelling it a stable personality trait (Saarijarvi, Salminen, \& Toikka, 2006). Alexithymia has been proven to have an adverse effect on relationship satisfaction, sexual satisfaction, and overall connected with a host of interpersonal difficulties (Humphreys, Wood, \& Parker, 2009).

The skin is the largest organ of the human body, yet cutaneous senses are often overlooked mediators of social interactions (Shut, et al., 2013). Touch is one of the primary elements for establishing attachment and it plays an important role in emotional communication, in evaluating the thoughts and feelings of others (Hertenstein, Verkamp, Kerestes, \& Holmes, 2006). "Simple touch," such as a light pressing, "protracted touch," for example a hug, and "dynamic touch," like caressing, are the three main categories of social touch (Morrison, Loken, \& Olausson, 2010). Subjects are generally able to differentiate between emotional categories based on how they have been touched (Shut, et al., 2013). They are able to distinguish anger, fear, disgust, love, gratitude, and sympathy at much-better-than-chance levels (Hertenstein, Keltner, App, Bulleit, \& Jaskolka, 2006). From a psychoanalytical point of view, the skin can be considered a central organ regarding border function, in the sense of protection and setting limits, and regarding sensory function, as the central contact organ (Grolle, et al., 2003).

Disgust is a feeling of revulsion arising as a response to an aversive stimulus that induces a motivation to withdraw from that stimulus (Rozin, Haidt, \& McCauley, 2000). Feelings of disgust are strongly related to and can be provoked by stimulation of the skin (Blake, Yih, Zhao, Sung, \& Harmon-Hones, 2016). The interpretation of such stimuli can be affected by the presence of skin diseases (Lahousen, et al., 2016). Apart from skin-related responses, disgust can be provoked by nutri- 
tional stimuli (Grolle, et al., 2003), and in more general terms unrelated to any particular stimuli, it can be regarded as the separate concepts of disgust sensibility and disgust sensitivity (van Overveld W. J., de Jong, Peters, Cavanagh, \& Davey, 2006).

Alexithymia has a proven and well documented relation to intimacy, relationship satisfaction, and interpersonal relationships, be it on a cognitive or emotional level. Evidence of its relationship to palpation perception, the integration of multisensory stimuli, and similar phenomena has also been documented. Physical touch, mediated by the skin, is naturally also a fundamental element of and integrally tied to interpersonal relationships, as it is one of the main mediators in conveying emotional information. Disgust is one of the many emotions closely related to skin perception, whether in healthy or pathological states, and represents a sensation that arises while withdrawing from contact with aversive stimuli. This paper is based on the conjecture that there is a relationship between alexithymia and perception of touch, be it at the level of intimacy or at the level of disgust, and it is the aim of this study to provide evidence in favor of this hypothesis.

\section{Methodology}

The research participants were medical sciences students. They completed a paper and pencil survey, part of which has been used for this research. A total of 357 students participated in the survey, 286 (82.9\%) were female, and the average participant age was 20.54 ( $\min =19$; $\max =26 ; \mathrm{SD}=1.24$ ). Apart from this demographical information, the survey consisted of the Skin Satisfaction Questionnaire (TSDQ-30), Toronto Alexithymia Questionnaire (TAS-20), and the Disgust Propensity and Sensitivity Questionnaire (DPSS).

The TAS-20 questionnaire is the most often used instrument to measure levels of alexithymia in both clinical and general populations (Timoney \& Holder, 2013). It consists of three subscales: factor 1 - difficulty identifying feelings (F1), factor 2 - difficulty describing feelings (F2), and factor 3 - externally oriented thinking (F3). The questionnaire was translated into Serbian (Trajanovic, Djuric, Latas, Milovanovic, Jovanovic, \& Djuric, 2013), and further validated on a large 
sample (Rokvic \& Jovanovic, 2018). The overall internal consistency of the TAS-20 in our study was on the lower end of acceptable margins $(\alpha=.78)$ and for the subscales, respectively ( $\alpha=.79, \alpha=.75, \alpha=52)$, with expectedly low values for the third factor (Rokvic \& Jovanovic, 2018).

The TSD-Q skin satisfaction questionnaire was initially developed in German (Kupfer, Brosig, Neimeier, \& Gieler, 2005) and further validated internationally (Shut, et al., 2013). The questionnaire consists of five subscales relating to touch sensitivity, shame, and disgust. These are: Touching self (Ts) $(\alpha=.84)$, Touched by parents (Tp) ( $\alpha=.803)$, Touched by partner (Tpa) ( $\alpha=.79)$, Disgust (Di) $(\alpha=.66)$, and Shame (Sh) (.62). The reliability of the touch related subscales is good, but those related to disgust and shame are somewhat diminished.

The DPSS questionnaire was devised to measure two different and separate aspects of disgust (van Overveld, et al., 2006). The first subscale measures disgust propensity (DP), irrespective of elicitors; in other words, how readily people respond with disgust. The disgust sensitivity (DS) subscale determines the degree of unpleasantness to which people experience disgust (van Overveld, de Jong, \& Peters, 2010). Both subscales demonstrated good internal consistency, with alpha values of .83 and .79 , respectively, and the same held true for the questionnaire as a whole, with an alpha value of 86 .

\section{Results}

Gender differences in scores were shown to exist in TAS-20 factor 3, externally oriented thinking, at the level of statistical significance $(\mathrm{p}=.001)$ in favor of men. There also registered detectable gender differences in the TSD-Q subscales of pleasure in touching parents, pleasure in touching partner, and shame at levels of statistical significance ( $\mathrm{p}=.001 ; \mathrm{p}<.001 ; \mathrm{p}=.017$ respectively) in favor of female participants. No significant correlations between participant age and any of the measured constructs were demonstrated. Correlation coefficients between measured constructs are represented in table 1. 
Table 1

Correlation coefficients between measured constructs.

\begin{tabular}{cccccccccccc}
\hline & $\begin{array}{c}\text { TAS- } \\
20\end{array}$ & F1 & F2 & F3 & Ts & Tp & Tpa & Di & Sh & DP & DS \\
\hline $\begin{array}{c}\text { TAS- } \\
20\end{array}$ & 1 & & & & & & & & & \\
F1 &, $81^{* *}$ & 1 & & & & & & & & & \\
F2 &, $83^{* *}$ &, $6^{* *}$ & 1 & & & & & & & & \\
F3 &, $49^{* *}$ &, 02 &, $16^{* *}$ & 1 & & & & & & & \\
Ts &, 09 &, $16^{* *}$ &, $12^{*}$ &,- 1 & 1 & & & & & & \\
Tp &,$- 13^{*}$ &,- 04 &,$- 11^{*}$ &,$- 14^{* *}$ &, 1 & 1 & & & & & \\
Tpa &,- 1 &, $1 *$ &,$- 11^{*}$ &,$- 26^{* *}$ &, $41^{* *}$ &, $34^{* *}$ & 1 & & & & \\
Di &, 09 &, $19^{* *}$ &, 1 &,$- 13^{*}$ &, $19^{* *}$ &, 08 &, $18^{* *}$ & 1 & & & \\
Sh &, $39^{* *}$ &, $41^{* *}$ &, $38^{* *}$ &, 00 & .01 &, 05 &, $11^{*}$ &, $19^{* *}$ & 1 & & \\
DP &, $16^{* *}$ &, $19^{* *}$ &, $2 * *$ &,- 07 &, $11^{*}$ &, $13 *$ &, $16^{* *}$ &, $41^{* *}$ &, $25 * *$ & 1 & \\
DS &, $199^{* *}$ &, $25^{* *}$ &, $15^{* *}$ &, 00 &, $14^{*}$ &, 1 &, $16^{* *}$ &, $18^{* *}$ &, $29 * *$ &, $57 * *$ & 1 \\
\hline
\end{tabular}

${ }^{*}$ Significant at the 0.01 level; ${ }^{*}$ Significant at the 0.05 level.

According to the criterion, participants were grouped into those with low alexithymia levels $(\mathrm{N}=232)$, medium levels $(\mathrm{N}=80)$, and high levels (N=45) (Parker, Taylor, \& Bagby, 2003). After conducting an ANOVA analysis grouping for alexithymia levels, differences in Tp were seen between those that have low and high alexithymia $(\mathrm{p}=.019)$, and in Sh between those with low and high alexithymia levels, as well as between those with low and medium levels $(\mathrm{p}<.001)$. Also, a difference was shown in disgust propensity between those with low and high alexithymia levels $(\mathrm{p}=.049)$ and in disgust sensitivity between those with low and medium alexithymia levels ( $\mathrm{p}=.005)$.

A regression model was created that measures the amount of alexithymia variance explained by other measured constructs. This was a three-step model. In Step 1, gender and age were controlled for; in Step 2, the Ts, Tp, Tpa, and Sh components of the TSD-Q scale were added; in Step 3 were added the Di, DP, and DS constructs. The results of the corresponding analyses are presented in table 3 . The individual contributions of constructs to the complete model, the model after Step 3, are presented in table 3 . 
Table 2

Step by step values of the regression model that show what part of alexithymia variance is explained by other measured constructs.

\begin{tabular}{llllll}
\hline & $\mathrm{r}^{2}$ & $\mathrm{r}^{2}$ change & Df & $\mathrm{F}$ & Sig. \\
\hline Step 1 & .009 & .009 & 2 & 1.602 & .203 \\
Step 2 & .221 & .212 & 6 & 16.507 & .000 \\
Step 3 & .234 & .013 & 9 & 11.747 & .000 \\
\hline Step 1
\end{tabular}

Step 1 - inclusion of age and gender into the model; Step 2 - inclusion of Ts, Tp, Tpa, and Sh into the model; Step 3 - inclusion of Di, DP, and DS into the model.

Table 3

Individual contributions of variables in the complete, step 3 , model.

\begin{tabular}{lll}
\hline & Beta & Sig. \\
\hline Gender & .074 & .134 \\
Age & -.095 & .049 \\
Touching self & .154 & .004 \\
Being touched by parents & -.119 & .02 \\
Being touched by partner & -.166 & .004 \\
Shame & .392 & .000 \\
Disgust & .007 & .901 \\
Disgust propensity & .054 & .384 \\
Disgust sensitivity & .081 & .171 \\
\hline
\end{tabular}

\section{Discussion}

The relatively small number of male participants presents some difficulties in interpreting findings along gender lines. Nevertheless, with regards to alexithymia, no significant differences were documented in overall TAS-20 score for F1 and F2, but only in F3. This is in opposition to general findings regarding alexithymia (Timoney \& Holder, 2013). Earlier studies examining the Serbian population indicated gender differences in F1, but not in F3 (Rokvic \& Jovanovic, 2018). This must be regarded as a peculiarity of the surveyed population. With regards to the TSD-Q instrument, cumulative scores from cross-cultur- 
al studies have confirmed gender differences in the disgust and shame subscales. While this study found differences in the shame subscale, differences in the subscales of regarding pleasure derived from touching self, parental touch, and partner touch also appeared. Additionally, no gender differences were demonstrated in the disgust subscale of the TSD-Q and in both individual subscales of the DPSS. This is in contrast with previous findings that show disgust as a function of gender, with female participants having significantly higher levels of disgust sensitivity (de Durschel \& Sherman, 1999). While these findings are somewhat puzzling, a logical possible explanation can be put forth. The sample consisted of medical sciences students, who are exposed to very specific clinical environments during their studies, encountering many situations that could be considered disgusting by the general population. The author of this paper could find no studies documenting disgust level differences between medical personnel and the lay population and its influence on gender differences in disgust sensitivity, but do believe that these new findings point to a possible new line of investigation regarding disgust sensitivity, vocation, and gender. Regarding gender differences in touch sensitivity, Shut et al. (2013) found cross-cultural differences in overall scores and gender differences. Without further data to draw upon, the author of this paper postulate that this study's findings represent a specific trait of the examined culture.

The results of this study show a weak positive correlation between F1, F2 and the Ts subscale, more strongly so between F1 and Ts. In contrast, the correlations between F1, F2 and Tp and Tpa are almost negligible and statistically insignificant. The results show that difficulty identifying feelings does not have an apparent relation with enjoyment; therefore, this is not a significant contributor to feeling pleasure or displeasure from physical touch, either from parents or from partners. Since there a weak positive correlation between touching self and difficulties describing feelings and identifying them was indicated, it could be postulated that there is a certain level of narcissism involved as a mediating trait, given the reports of a correlation between alexithymia and narcissism (Jonason \& Krause, 2013). On the other hand, externally oriented thinking as a facet of alexithymia, described as pragmatic thinking, lower imagination, and attributing lower value to emotions, 
correlates especially negatively with $\mathrm{Tp}$ and Tpa. That this negative correlation was more pronounced regarding deriving pleasure from being touched leads the author to believe that alexithymic persons simply find less value in such relations, as they possibly do not consider them pragmatic for the furthering of their relationship and apparently possess a certain aversion from it. This opens up interesting questions and possibilities for further investigations and interpretations of the role of alexithymia in the establishing, maintaining, and quality of intimate relations.

A low correlation was found between disgust and the overall alexithymia score, more precisely F1 and F2, while no significant correlation with F3 was apparent. As disgust has been defied as an impulse to withdraw from revolting stimuli, such findings may indicate that there is a certain amount of disgust-related withdrawal from emotional stimuli that cannot be described or identified. The exact relationship and impact of disgust in this matter has been proven difficult to determine, as in the regression model employed by this study all three measured disgust variables accounted for only $1.3 \%$ of the variance. Perhaps examining different facets of disgust and alexithymia itself might yield different results.

Shame has been well documented to be associated with alexithymia (Franzoni, et al., 2013, Suslow, Donges, Kersting, \& Arlot, 2000), but the author found most of the research has been carried out on clinical populations, especially those with eating disorders and distorted body image. Their findings reflect those found here, which indicate a moderate correlation between the overall TAS- 20 score, F1, and F2 with shame. Earlier findings also indicate a low correlation between F3 and shame, yet this weak linkage is even more pronounced in this study's findings, with no correlation established whatsoever. The results show that relations between shame and alexithymia are constant weather it is body image, shame, or skin-satisfaction-related shame. To further underscore this relation, shame was observed as the single strongest contributing variable to the employed regression model, covering $39.2 \%$ of the variance explained by the model.

After performing ANOVA, it was found that there are differences between levels of measured constructs when grouped by levels of 
alexithymia. Differences regarding satisfaction with touching self were documented between those with low and high alexithymia levels. Significant differences in shame levels between all three groups (low, regular and high alexithymia) were also demonstrated. These differences are also present when grouping disgust propensity and sensitivity by alexithymia levels. These findings further underscore the results of correlation analysis.

The regression model created for this study attempted to determine the amount of alexithymia variance explained by other measured constructs. After controlling for age and gender, it was found that touch satisfaction and shame facets explain $21.2 \%$ of the variance, with disgust subscales explaining only $1.3 \%$. All skin satisfaction subscales have a unique contribution to the final model. The author had been expecting a stronger contribution by the disgust subscales given the correlation coefficients' theoretical implication, but this was simply not the case in this sample and, as stated before, the author believe that further research into the nature of the relationship between various facets of disgust and alexithymia is warranted.

\section{Conclusion}

Amongst skin satisfaction parameters, alexithymia is most strongly related with shame. Disgust parameters appear to have a very weak influence on alexithymia variance. Although skin is one of the primary sense organs and touch is capable of conveying emotion, there is only a weak correlation between, and measured influence of, satisfaction from skin and alexithymia. Further research into this area should concentrate on determining the influence of other skin related sensations, beyond satisfaction and disgust, on alexithymia, or vice versa, in order to reach a more complete understanding of this complex condition. 


\section{Literature}

Bermond, B., Vorst, H. C., Vingerhoets, A. J., \& Gerritsen, W. (1999). The Amsterdam Alexithymia scale: Its psychometric values and correlations with other personality traits. Psychotherapy and Psychosomatics, $68,241-51$.

Blake, K. R., Yih, J., Zhao, K., Sung, B., \& Harmon-Hones, C. (2016). Skin-transmited pathogenes and the heebie-jeebies: evidence for the subscaless of disgust stimuli that evoke a qualitatively unique emotional response. Journal of Cognition and Emotion, 31(6), 1153-68.

de Durschel, B., \& Sherman, M. F. (1999). Disgust sensitificy as a function of the Big Five and gender. Personality and Individual Differences,26(4), 739-748.

Franzoni, E., Gualandi, S., Caretti, V., Schmmenti, A., Di Petro, E., Pelegrini, G., ... Pellicciari, A. (2013). The relationship between alexithymia, shame, trauma and body image dissorders: investigation over a large clinical sample. Neuropsychiatric Disease and Treatment, 9, 185-93.

Frewen, P. A., Dozolis, D. J., Neufeld, R. W., \& Lanius, R. A. (2008). Meta-analysis of alexithymia in posttraumatic stress disorder. Journal of Traumatic Stress, 21(2), 243-6.

Grolle, M., Kupfer, J., Brosig, B., Neimeier, V., Henninghausen, L., \& Gieler, U. (2003). The Skin satisfaction Questionnaire - an Instrument to Assess Attitudes toward the Skin in Healry Persons and Patients. Dermatology + Phychosomatics, 4(1), 14-20.

Grynberg, D., \& Pollatos, O. (2015). Alexithymia modulates the expirience of the rubber hand illusion. Frontiers in Human Neuroscience. https:// doi.org/10.3389/fnhum.2015.00357

Grynberg, D., Corneille, O., Maurage, P., Vermeulen, N., Bertholz, S., \& Luminet, O. (2012). Alexithymia and the processing of Emotional Facial Expressions (EFEs): Systemic Review, Unanswered Questions and Further Perspectives. PLOSone, 7(8), https://doi.org/10.1371/ journal.pone.0042429.

Grynberg, D., Davydov, D. M., Vermeulen, N., \& Luminet, O. (2012). Alexithymia is associated with a augmenter profile, but not only: evidence for anticipation to arousing music. Scandinavian Journal of Psychology, 53, 375-81.

Hertenstein, M. J., Keltner, D., App, B., Bulleit, B. A., \& Jaskolka, A. R. (2006). Touch Communicates Distinct Emotions. Emotion, 6(3), 528-33.

Hertenstein, M. J., Verkamp, J. M., Kerestes, A. M., \& Holmes, R. M. (2006). The communicative function of touch in humans, non-human primates and rats: A review and synthesis of the empirical research. General Psychology Monographs, 1, 5-94. 
Honkalampi, K., Hintikka, J., Tanskanen, A., Lehtonen, J., \& Viinamaki, H. (2000). Depression is strongly associated with alexithymia in the general population. Journal of Psychosomatic Research, 48(1), 99-104.

Humphreys, T. P., Wood, L. M., \& Parker, J. A. (2009). Alexithymia and satisfaction in intimate relationships. Personality and Individual DIfferences, 46(1), 43-7.

Jonason, P. K., \& Krause, L. (2013). The emotional deficites associated with the Dark Triad traits: Cognitive empathy, affective empathy and alexithymia. Personality and Individual Differences, 55(5), 532-7.

Koponen, S., Taiminen, T., Honkalampi, K., Joukamaa, M., Viinamaki, H., \& Kurki, T. (2005). Alexithymia after traumatic brain injury: Its relation to magnetic resonance imaging findings and psychiatric disorders. Psychosomatic Medicine, 67(5), 807-12.

Kupfer, J., Brosig, B., Neimeier, V., \& Gieler, U. (2005). Validity of the Touch-Shame-Disgust-Questionnaire (TSD-Q). Psychotherapie, Psychosomatik, Medizinishe Psychologie, 55, 139.

Lahousen, T., Kupfer, J., Gieler, U., Hofer, A., Linder, D. M., \& Shut, C. (2016). Difference Between Psoriasis Patients and Skin-healthy controles Concerning appraisal of Touching, Shame and Disgust. Acta Dermatovenerologica, 96,78-82.

Mattila, A. K., Poutanen, O., Koivisto, A.-M., Salokanges, R. K., \& Joukamaa, M. (2007). Alexithymia and Life Satisfaction in Primary Healthcare Patients. Psychosomatics, 48(6), 523-9.

Morrison, I., Loken, L. S., \& Olausson, H. (2010). The skin as a social organ. Experimental Brain Research, 204(3), 305-14.

Parker, J. D., Taylor, G. J., \& Bagby, M. R. (2003). The 20-Item Toronto Alexithymia Scale: III. Reliability and factorial validity in a community population. Journal of Psychosomatic Research, 55(3), 269-75.

Rokvic, N., \& Jovanovic, T. (2018). Alexithymia measured with the TAS-20 questionnaire: Determining the validity of the factor structure and its relation to life satisfaction and the Big Five personality traits. Psiholoska istrazivanja, 21(1), 23-40.

Rozin, P., Haidt, J., \& McCauley, C. R. (2000). Disgust. In M. Lewis, \& J. M. Haviland-Jones, Handbook of Emotions (pp. 637-653). New York: Guilford Press.

Saarijarvi, S., Salminen, J. K., \& Toikka, T. (2006). Temporal stability of Alexithymia Over a Five-Year Period in Outpatients with Major Depression. Psychotherapy and Psychosomatics, 75, 107-12.

Shut, C., Linder, D., Brosig, B., Neimeir, V., Ermler, C., Madejski, K., ... Kupher, J. (2013). Appraisal of touching behavior, shame and disgust: a cross-cultural-study. International Journal of Culture and Mental Health, 6(1), 1-15. 
Sivik, T. (1993). Alexithymia and Hypersensitivity to Touch and Palpation. Integrative Physiological and Behavioral Science, 28(2), 130-6.

Suslow, T., Donges, U.-S., Kersting, A., \& Arlot, V. (2000). 20-item Toronto Alexithymia Scale: Do difficulties describing feelings assess proneness to shame instead of difficulties symbolizing emotion? Scandinavian Journal of Psychology, 44(4), 329-34.

Taylor, G. J. (1984). Alexithymia: concept, measurement and implication for treatment. The American Journal of Psychiatry, 141(6), 725-32.

Timoney, L. R., \& Holder, D. M. (2013). Emotional Processing Deficites and Happiness: Assessing the Measurement, Correlates and Well-Being of People with Alexithymia. London: Springer.

Trajanovic, N. N., Djuric, V., Latas, M., Milovanovic, S., Jovanovic, A. A., \& Djuric, D. (2013). Serbian Translation of the 20-Item Toronto Alexithymia Scale: Psychosomatic Properties and the New Methodological Approach in Translating Scales. Srpski arhiv za celokupno lekarstvo, 141(5-6), 366-70.

van Overveld, M., de Jong, P. J., \& Peters, M. L. (2010). The Disgust Propensity and Sensitivity Scale - Reviseted: Its predictive value for avoidance behavior. Personality and Individual Differences, 49(7), 706-11.

van Overveld, W. J., de Jong, P. J., Peters, M. L., Cavanagh, K., \& Davey, G. C. (2006). Disgust propensity and disgust sensitivity: Separate constructs that are differentially related to specific fears. Presonality and Individual Differences, 41(7), 1241-52.

Westwood, H., Kerr-Gaffney, J., Stahl, D., \& Tchanturia, K. (2017). Alexithymia in eating disorders: Systemic review and meta-analyses of studies using the Toronto Alexithymia Scale. Journal of Psychosomatic Research, 99, 61-81. 